\title{
Médiévales
}

Langues, Textes, Histoire

72 | printemps 2017

Roman du Genji et société aristocratique au Japon

\section{Les concours de poèmes comme rituels de cour. Autour du « Concours de poèmes tenu au palais impérial la quatrième année de l'ère Tentoku [960]» (Tentoku yonen dairi uta awase)}

Poetry Competitions as Court Rituals. Form and Function of the "Imperial Palace Poetry Competition of the Fourth Year of Tentoku", Tentoku yonen dairi uta awase (960)

Michel Vieillard-Baron

\section{OpenEdition} Journals

Édition électronique

URL : https://journals.openedition.org/medievales/8072

DOI : $10.4000 /$ medievales.8072

ISSN : 1777-5892

Éditeur

Presses universitaires de Vincennes

Édition imprimée

Date de publication : 22 juin 2017

Pagination : 73-86

ISBN : 978-2-84292-612-0

ISSN : 0751-2708

Référence électronique

Michel Vieillard-Baron, «Les concours de poèmes comme rituels de cour. Autour du « Concours de poèmes tenu au palais impérial la quatrième année de l'ère Tentoku [960] » (Tentoku yonen dairi uta awase) », Médiévales [En ligne], 72 | printemps 2017, mis en ligne le 28 février 2019, consulté le 23 avril 2022. URL : http://journals.openedition.org/medievales/8072 ; DOI : https://doi.org/10.4000/ medievales.8072 
Michel Vieillard-Baron

\title{
Les concours de poèmes comme rituels de cour
}

\author{
Autour du "Concours de poèmes tenu au palais \\ impérial la quatrième année de l'ère Tentoku [960] " \\ (Tentoku yonen dairi uta awase)
}

L'empereur Murakami (926-967; r. 946-967) fut un souverain particulièrement sensible à la poésie. En 951, il fit établir au palais un Bureau de la poésie (waka dokoro) chargé, d'une part, de compiler son anthologie impériale, le Recueil de poèmes sélectionnés postérieurement $(\text { Gosen wakash û })^{1}$ et, d'autre part, d'établir la lecture des 4500 pièces du Man.yôsh û, somme de la poésie antique achevée vers $759^{2}$. Il commanda également un important concours de poèmes (uta awase), manifestation qui va nous occuper ici.

Les concours de poèmes consistaient à faire s'affronter deux équipes, appelées « équipe de gauche» (hidari kata) et «équipe de droite» (migi $k a t a)$, qui devaient produire des waka - c'est-à-dire des poèmes de trente et une syllabes en japonais ${ }^{3}-$, sur des sujets distribués à l'avance (kendai) ou sur place (sokudai ou tôza). Le jour du concours, les poèmes de l'équipe de gauche étaient appariés à ceux de l'équipe de droite et déclamés par un

1. L'empereur Daigo avait ordonné en 905 la compilation de la première anthologie impériale de poésie japonaise, le Recueil de poèmes anciens et modernes (Kokin wakashû) ; celle de l'empereur Murakami est la seconde (d'où son titre de recueil de poèmes sélectionnés postérieurement [au Kokin wakashî]). Vingt et une anthologies de ce type furent produites, la dernière au $X^{\mathrm{e}}$ siècle. Elles étaient considérées comme le symbole du règne éclairé du souverain et à ce titre extrêmement prestigieuses.

2. Les poèmes du Man.yôshû (dont le titre peut signifier «Recueil des dix mille feuilles » ou des « dix mille règnes ») sont notés à l'aide d'un système d'écriture extrêmement complexe constitué exclusivement de caractères chinois - le Japon ne disposait pas de système d'écriture propre -, pris soit pour leur valeur sémantique, soit pour leur valeur phonétique (les poèmes étant lus en pur japonais). Ce système d'écriture était devenu au $\mathrm{X}^{\mathrm{e}}$ siècle difficilement lisible, d'où l'initiative de l'empereur Murakami. Cf. Man.yôshû. Livres I-XX, 5 vol., trad. R. SIEFFERT, Paris, 1997-2003.

3. On composait également au Japon des poèmes en chinois (kanshi). Comme nous allons le voir, les poèmes en chinois firent eux-mêmes l'objet de concours. 
lecteur (kôji). Chaque paire de poèmes (un poème de l'équipe de gauche et un autre de l'équipe de droite), composés sur le même sujet (dai), constituait une manche (ban). Un arbitre (hanja), choisi parmi les poètes faisant autorité, était chargé de départager les pièces. Il justifiait son choix dans des attendus de jugement (hanji), parfois très détaillés. L'équipe gagnante était celle dont le plus grand nombre de poèmes avaient été déclarés victorieux. Le plus ancien exemple conservé de ce type de manifestation date de $885^{4}$. Les procès-verbaux de plus de 450 de ces concours ont été conservés - intégralement ou sous forme de fragments - pour la période allant du $\mathrm{IX}^{\mathrm{e}}$ au XIII $\mathrm{e}^{\mathrm{e}}$ siècle. Leur examen révèle une extraordinaire variété : le plus petit qui se soit tenu compte une seule manche, soit deux poèmes ${ }^{5}$; le plus important, six cents manches (soit mille deux cents waka en lice) ${ }^{6}$. Ces concours pouvaient se dérouler au palais impérial, dans des résidences privées, des temples, des sanctuaires, ou encore n'exister que sur le papier ${ }^{7}$. Certains concours ont été arbitrés par un seul arbitre, d'autres par plusieurs, voire collectivement (les participants prenant part à l'évaluation). Il existe également quelques exemples de concours dont les poèmes n'ont fait l'objet d'aucune évaluation. Bref, les cas de figure sont multiples ${ }^{8}$.

Les concours de poèmes peuvent être abordés de différentes façons. On a souvent étudié leur rôle dans le développement de la composition poétique sur sujet imposé (dai.ei). En effet, pour être départagés, les poèmes devaient traiter le même thème ${ }^{9}$. Leur rôle dans l'approfondissement de la réflexion poétique - les arbitres devant justifier leurs choix - a également été souligné ${ }^{10}$. C'est sous un angle tout à fait différent que nous les examinerons ici : il s'agira de mettre en relief l'aspect rituel des concours de poèmes les plus officiels, ceux qui se déroulaient au palais impérial. En examinant

4. Il s'agit du Minbu-kyô ke uta awase ou «Concours de poèmes chez le chef du département de la population » qui se déroula chez Ariwara no Yukihira (818-893).

5. Il s'agit du Masaki no musumetachi uta awase ou «Concours de poèmes des filles de Fujiwara no Masaki » qui eut lieu vers 985.

6. Il s'agit du Roppyaku ban uta awase ou « Concours de poèmes en six cents manches » commandé par Fujiwara no Yoshitsune (1169-1206) et achevé vers 1194.

7. Ainsi le Sengohyaku ban uta awase ou «Concours de poèmes en mille cinq cents manches », soit 3000 poèmes en lice, qui fut commandé par l'empereur Gotoba et achevé vers 1202 sans que les poètes se soient réunis (tout fut réglé par écrit).

8. Pour un bref rappel de l'histoire des concours de poèmes, voir J. PIGEOT, Michiyukibun. Poétique de l'itinéraire dans la littérature du Japon ancien, Paris, 2009, p. 76-83 ; ainsi que ITO S., «The Muse in Competition : Uta-awase Through the Ages », Monumenta Nipponica, 37/2 (1982), p. 201-222. Signalons qu'on doit à cette dernière un recueil de concours de poèmes traduits en anglais : An Anthology of Traditional Japanese Poetry Competitions. Uta-awase (913-1815), Bochum, 1991.

9. La composition sur sujet imposé devint majoritaire à partir du XII siècle.

10. Sur ces points, voir par exemple notre ouvrage, Fujiwara no Teika (1162-1241) et la notion d'excellence en poésie. Théorie et pratique de la composition dans le Japon classique, Paris, 2001. 
leur minutieuse mise en scène et la somptuosité des décors, nous essaierons de déterminer leur fonction au sein de la vie de cour. Pour illustrer notre propos, nous étudierons le «Concours de poèmes tenu au palais impérial la quatrième année de l'ère Tentoku [960] » (Tentoku yonen dairi uta awase), titre abrégé désormais en «Concours de Tentoku ». Cette manifestation est importante à plusieurs titres. Tout d'abord, les règles et le protocole définis pour ce concours servirent pour les manifestations de ce type organisées par la suite : il s'agit donc en quelque sorte d'un modèle. Deuxièmement, cinq relations (nikki) du concours ont été conservées, ce qui est tout à fait exceptionnel : celle de l'empereur en personne (A), celle d'un membre de la chancellerie privée ${ }^{11}$, non identifié (B) - toutes deux écrites en chinois, la langue de l'administration et de la littérature en prose « sérieuse » ${ }^{12}-$ les trois autres relations, rédigées en langue vernaculaire, sont dues pour deux d'entre elles à des dames du palais ayant pris part à la manifestation, non identifiées $(\mathrm{C}$ et $\mathrm{E})$, et pour la troisième, à un homme - un fonctionnaire de cour ? - également non identifié (D) ${ }^{13}$. Afin de comprendre comment se déroulait ce type de manifestations nous traduirons intégralement la relation de l'empereur que nous complèterons grâce aux informations fournies par les quatre autres comptes rendus. Dans un second temps, nous comparerons la relation de l'empereur avec les règles pour les concours se déroulant au palais impérial telles que les a définies, deux siècles plus tard, le poète et poéticien Fujiwara no Kiyosuke (1104-1177) dans un important traité.

\section{Le concours de Tentoku}

Avant de lire ces textes, rappelons brièvement les données concernant notre concours. Le « Concours de poèmes de l'ère Tentoku » fut commandé par l'empereur Murakami, qui écrit dans sa relation (A) :

Le 30 du troisième mois de l'année Tentoku 4 [960], jour Terre cadet-serpent, s'est tenu un concours de poèmes des dames du palais. L'automne de l'année précédente, au huitième mois, des courtisans à mon service avaient procédé

11. Signalons que, dans cet article, la titulature est traduite suivant F. HÉRAIL, La Cour et l'administration du Japon à l'époque de Heian, Genève, 2006.

12. Sur l'usage de l'écriture en chinois et en langue vernaculaire dans le Japon classique, voir F. HÉRAIL, « Lire et écrire dans le Japon ancien », dans V. ALLETON éd., Paroles à dire. Paroles à écrire, Paris, 1997, p. 253-274.

13. Pour toutes ces relations et le procès-verbal du concours, nous nous fondons sur le texte établi par HaGiTANi B., recueilli dans Uta awase shû, coll. Nihon Koten Bungaku Taikei, vol. 74, Tôkyô, 1965 (rééd. 1979), p. 78-104. La relation de l'empereur, citée ici et dans la suite de l'article, figure p. 88-94 de cette édition. Signalons que notre concours est également important d'un point de vue strictement poétique, aspect que nous ne pouvons pas aborder ici, faute de place. Voir à ce sujet TERADA S., Figures poétiques japonaises. La genèse de la poésie en chaîne, Paris, 2004, p. 227-229 et 260-261. 
à un concours de leurs poésies écrites en chinois. À cette occasion, l'adjointe du service intérieur ainsi que des dames du cinquième rang, entre autres, me dirent : «Puisque les hommes mettent en lice leurs compositions en chinois, les femmes devraient faire un concours de poèmes japonais [waka]». Et c'est ainsi que durant le deuxième mois de cette année [960] furent arrêtés les membres de l'équipe de gauche et de droite. Parmi ceux-ci figuraient les «dames du vestiaire [c.-à-d. des épouses de l'empereur] » $[k o \hat{i}]^{14}$, Fujiwara no Shûshi et Fujiwara no Yûjo, qui ont été nommées respectivement chef de l'équipe de gauche et de droite. J'ai donné l'ordre [aux poètes des équipes] de composer et de sélectionner les meilleures pièces [...].

Cette citation nous apprend que le concours fut ordonné à l'instigation des dames du palais et qu'il s'agissait d'un concours de poèmes sélectionnés (senka awase), c'est-à-dire constitué des meilleurs poèmes produits pour l'occasion. Il convient de préciser que, si seulement douze poètes parmi les meilleurs du temps composèrent et prirent part au concours proprement dit (huit pour l'équipe de gauche, dont deux femmes) et quatre pour celle de droite (dont une femme), chacune des équipes comportait 39 membres, dames du palais, hauts dignitaires et courtisans, soit un total de 78 participants auxquels il convient d'ajouter les dix musiciens. L'empereur, de par sa fonction, dominait l'ensemble des participants et constituait le centre de gravité autour duquel toute la manifestation s'organisait. Douze sujets furent imposés aux poètes (parmi lesquels «brume», « fauvette », « glycines », associés au printemps, « coucou » et «deutzies en fleurs ${ }^{15}$ » associés à l'été) : ils illustrent le passage du printemps à l'été, ce qui est parfaitement en accord avec le moment où s'est tenu le concours (le dernier jour du printemps et l'aube du premier jour d'été selon le calendrier alors en vigueur). L'arbitre était le ministre de gauche ${ }^{16}$, Fujiwara no Saneyori (900-970), réputé pour son talent poétique, tant en chinois qu'en japonais : lorsque des poèmes étaient difficiles à départager, il sollicitait pour trancher l'avis du souverain.

Dans sa relation l'empereur écrit :

[J'ai ordonné ce concours] car je déplorais que la Voie des Lettres [puisse] se déliter et s'interrompre. Dans les temps à venir, il se trouvera sans doute des gens qui, ignorant mes intentions, répandront des calomnies, disant que

14. Les empereurs pouvaient avoir plusieurs épouses de différentes catégories: « impératrice » (chûgû ou kôgô), «épouse impériale » (nyôgo), « dame du vestiaire » (kôi) et « dame de la chambre » (miyasundokoro). Cf. F. HÉRAIL, La Cour et l'administration du Japon..., p. 499-502.

15. Arbustes dont les fleurs blanches, groupées en épis, s'épanouissent en mai-juin :

Cf. C. PÉronny, Les Plantes du Man.yô-shû, Paris, 1993, p. 191.

16. Il existait également une fonction de ministre de droite. 
tout cela fut motivé par goût pour une vaine magnificence, qu'il s'agissait d'une passion secrète et exclusive.

Le souverain inscrit donc ce concours dans une démarche visant à préserver la littérature - la poésie en particulier - dont il se désigne implicitement comme le protecteur. Dans sa relation en langue vernaculaire $\mathrm{C}$, une femme participant au concours (non identifiée) écrit :

Le 2 du troisième mois, l'adjointe du service intérieur présenta aux chefs de chacune des équipes, dans le bureau des dames du palais, la liste des membres de leur équipe. Celle-ci avait été établie par l'empereur en personne le 29 du deuxième mois.

Cette femme précise en outre :

Le 3 [du troisième mois], les sujets furent distribués. C'est la dame adjointe du service intérieur qui les a notés devant sa majesté ${ }^{17}$.

Nous comprenons ainsi que les préparatifs du concours commencèrent à la fin du second mois et que l'empereur joua un rôle central dans la composition des équipes et dans le choix des thèmes poétiques.

Pour la tenue du concours, un espace fut spécialement aménagé : il comprenait la galerie extérieure [en] bordant à l'ouest ${ }^{18}$ le pavillon de Pureté et de Fraîcheur [Seiryôden], où se déroulait la vie quotidienne du souverain, ainsi que la galerie extérieure Est du pavillon Arrière [Kôryôden] ${ }^{19}-$ les deux galeries se faisaient face. La large galerie de passage couverte qui, située au milieu de la galerie ouest et de la galerie est, reliait les deux pavillons, fut également mobilisée, ainsi que les deux cours closes [tsubo], plantées de végétaux, qui se trouvaient au nord et au sud de la galerie de passage $^{20}$. L'empereur écrit dans sa relation :

17. Hagitani B.éd., Uta awase shû, p. 93-94.

18. TAKIGAWA K., "Gishiki no ba to waka no chii » (dans Waka wo rekishi kara yomu, Tôkyô, 2002, p. 71-87), voit dans le fait que la manifestation se soit déroulée sur la galerie Ouest un signe indiquant que ce concours, organisé à l'instigation des dames du palais, était considéré à l'origine comme une manifestation privée. Il fut néanmoins très tôt considéré comme le modèle des concours officiels.

19. Le pavillon Arrière était considéré comme le prolongement du pavillon de Pureté et de Fraîcheur.

20. Faute de place, il n'est pas possible d'inclure ici de plan : nous renvoyons à l'ouvrage de N. FIÉvÉ éd., Atlas historique de Kyôto, Paris, 2008, p. 67 et 68, où l'on trouvera un plan du palais impérial et des pavillons mentionnés. Voir également M. MAURIN, «Sur les pas du Genji : le palais de Heian », Cipango. Cahiers d'études japonaises, numéro hors-série, Autour du Genji monogatari, 2008, p. 186-213 (https://cipango.revues.org/601). 
Voilà de quelle manière s'est déroulé [le concours de poèmes] : de la large galerie centrale couverte reliant le pavillon de Pureté et de Fraîcheur au pavillon Arrière, on a provisoirement enlevé les volets situés vers le Nord et on a installé à l'intérieur de cette galerie les places pour les hauts dignitaires. Les places pour les membres des équipes de gauche et de droite ont quant à elles été installées sur la galerie extérieure est du pavillon Arrière, l'équipe de gauche au sud, l'équipe de droite, au nord. Les dames ont été séparées [des hommes] et placées dans un espace délimité par des stores situé sur la galerie ouest du pavillon de Pureté et de Fraîcheur. C'est également sur cette galerie, au niveau de la cinquième travée [ $\mathrm{ma}$ ], qu'a été placé [mon] fauteuil. On a utilisé le fauteuil [habituellement rangé] dans la salle des plateaux ${ }^{21}$ et on a relevé les stores de [ma] travée.

Comme le révèle ce passage, l'emplacement des participants au concours était réglé principalement selon deux critères : leur rang et leur sexe. Les hauts dignitaires sont les hommes placés le plus près de l'empereur, sur la galerie de passage qui débouche en face du siège du souverain. Le souverain est le seul à disposer d'un fauteuil, tous les autres participants sont assis sur un coussin ou une natte disposés à même le sol. Les femmes de haute condition sont, conformément aux usages du temps, placées dans un espace où elles demeureront invisibles aux regards des hommes (espace jouxtant la travée de l'empereur), au nord (pour les femmes de l'équipe de droite) et au sud (pour celles de l'équipe de gauche). Nous savons que les stores baissés de leur espace furent en outre doublés d'écrans afin d'assurer une opacité maximale ${ }^{22}$. Ces femmes participaient donc au concours mais ne pouvaient pas voir son déroulement. Nous savons également que les membres de l'équipe de gauche portaient des tenues rouges et celles de l'équipe de droite, des tenues vertes ${ }^{23}$.

L'empereur continue :

À l'heure du Singe [vers 16 heures],j'ai pris place sur mon siège. Après un temps assez long, les membres de l'équipe de droite sont entrés par le nord et $\mathrm{m}$ 'ont présenté leur « grève des immortels » $[\text { suhama }]^{24}$ avec les poèmes ;

21. Où l'on mettait la dernière main aux repas de l'empereur.

22. $C f$. la relation C dans HaGitani B. éd., Uta awase sh $\hat{u}$, p. 102.

23. $C f$. les relations $\mathrm{B}, \mathrm{C}$ et $\mathrm{E}$.

24. Charlotte Von Verschuer nous rappelle (communication personnelle) : la «grève des immortels» (suhama) se présentait sous forme d'une table présentoir garnie d'une «création» (tsukurimono) représentant une plage et une montagne, avec des rochers, des végétaux et des oiseaux, notamment des grues. Le décor évoquait le mont Hôrai, situé dans une île lointaine habitée par les «immortels » selon la tradition taoïste de la Chine antique. Il était constitué de matières précieuses, entre autres de l'or, de l'argent, des bois exotiques dont l'aloès - à ne pas confondre avec la plante grasse du même nom - et des parfums rares comme le musc. Ce type de « création » a connu une grande vogue à la cour japonaise à partir 
il s'agissait d'une table en bois d'aloès de la meilleure qualité avec pieds recourbés vers l'extérieur sculptés de motifs floraux [posée sur] une table de dessous en bois odorant léger. L'étoffe qui recouvrait [ « la grève des immortels »] [ohohi] était en sergé de soie à motifs de fleurs, brodé de fleurs, de saules et d'oiseaux ; [l'ensemble était posé sur] un tapis de soie bleue avec motifs tissés. Quatre fillettes, au service de la «dame du vestiaire » chef de l'équipe [de droite], m'ont apporté cette "grève des immortels » et se sont placées sur la galerie de passage, en face de l'endroit où je me trouvais. La « grève des immortels » pour les « baguettes de victoire » $[k a z u$ sashi] ${ }^{25}$ fut posée dans la cour [close] nord, devant le coussin rond du jeune suivant préposé au «baguettes de victoire».

L'empereur arrive donc le premier sur les lieux où va se dérouler le concours. L'équipe de droite lui présente ses compositions disposées sur une « grève des immortels ». Remarquons que dans la tradition de cour la gauche avait préséance sur la droite. C'est donc l'équipe de gauche qui aurait dû présenter ses poèmes la première, mais le retard de cette équipe ${ }^{26}$ fit qu'elle dut céder sa place. Chaque équipe disposait de deux «grèves des immortels », une pour les poèmes, l'autre pour les « baguettes de victoire ». L'empereur poursuit :

Après un long moment, l'équipe de gauche est arrivée par le Sud en passant par la salle des courtisans en service [sise sur la galerie extérieure du pavillon de Pureté et de Fraîcheur] et m'a présenté sa "grève des immortels » avec les poèmes. Il s'agissait d'une table en bois de santal rouge de la meilleure qualité avec pieds recourbés vers l'extérieur sculptés de motifs floraux [posée sur] une table de dessous en bois de sappan. L'étoffe qui recouvrait [《la grève des immortels »] $]^{27}$ était en sergé de soie à motifs de fleurs sur lequel on avait brodé [des poèmes] notés en «écriture en forme

du IX ${ }^{\mathrm{e}}$ siècle, notamment après l'intronisation de l'empereur Junna (r. 823-833) où il faisait partie du décor protocolaire de bon augure pour les Grandes prémices impériales, le banquet du Nouvel An ou les banquets de mariage, etc. Ces plateaux-présentoirs étaient utilisés pour exposer les cadeaux ou les objets destinés à un invité de marque. En 1008, c'est le repas de l'empereur qui y avait été déposé à l'occasion de la commémoration des cent premiers jours du prince Atsuhira.

25. Ustensiles utilisés pour comptabiliser les victoires de chacune des équipes. Nous savons grâce à la relation $\mathrm{B}$ que les baguettes de victoire de l'équipe de droite, faites d'or et d'argent, avaient la forme de rameaux de saule et de corète, celles de l'équipe de gauche, également en or, avaient la forme de fleurs de glycine.

26. $C f$. la relation E, dans Hagitani B. éd., Uta awase shû, p. 101.

27. Ce tissu qui protégeait et cachait l'arrangement de la «grève des immortels » était probablement ôté au moment où débutait le concours. 
de roseau » ashide $^{28}$; [l'ensemble était posé] sur un tapis de soie violette. Six fillettes au service de la «dame du vestiaire» [chef de l'équipe de gauche] sont apparues en le portant et l'ont déposé [devant moi] comme l'avait fait l'équipe de droite. La « grève des immortels » pour les baguettes de victoires [de l'équipe de gauche] a été posée devant le jeune suivant préposé à leur maniement, dans la cour [close] Sud. Dans un premier temps les fillettes avaient placé la « grève des immortels » sous la table, mais elles l'ont ensuite placée correctement.

Chacune des équipes présente de la même manière au souverain sa « grève des immortels » avec les poèmes. Seuls varient quelques détails : entre autres, la direction d'entrée, le nombre de fillettes portant les tablesprésentoirs, la décoration de celles-ci ${ }^{29}$ et la couleur des étoffes.

Ordre fut ensuite donné aux hauts dignitaires de prendre place. C'est ainsi qu'ont pris place le ministre de gauche [Fujiwara no Saneyori], alias Seishinkô, le grand conseiller Minamoto no Takaakira, le général de la garde du corps section de droite Fujiwara no Morotada, les auditeurs [Minamoto no] Masanobu et [Fujiwara no] Asanari. Ensuite, les courtisans à mon service (jishin) de chacune des équipes ont pris place. À ce moment la nuit était déjà tombée ; aussi apporta-t-on des lampes et des torchères. Les torchères furent mises en place dans les cours [closes] Sud et Nord. On fit ensuite entrer les personnes qui devaient lire les poèmes. Pour l'équipe de gauche, c'est le commandant de la garde des gendarmes, section de droite [Minamoto no] Nobumitsu ; pour l'équipe de droite, le général en second de la garde du corps section de droite [Minamoto no] Hiromasa. Ces derniers se sont approchés des «grèves des immortels » et ont lu les poèmes. Ceux de l'équipe de gauche étaient écrits sur les feuilles d'un rameau de corète aux fleurs [faites d']or ; ceux de l'équipe de gauche sur des feuillets miniature de papiers à poèmes [shikishi]. Le général en second section de gauche Koresada et le général en troisième de la garde du corps section de droite Sukenobu les éclairaient à l'aide de torches. Pendant ce temps, les jeunes suivants, installés dans les cours [se tenaient prêts à] piquer les baguettes de victoires. Comme je lui en ai donné l'ordre, le ministre de gauche [Minamoto no] Saneyori a départagé les poèmes.

28. Sur ce style calligraphique qui consistait à déformer les signes d'écriture de manière à évoquer des roseaux, des rochers ou des oiseaux, $C f$. C.-A. BRISSET, À la croisée du texte et de l'image. Paysages cryptiques et poèmes cachés (ashide) dans le Japon classique et médiéval, Paris, 2009.

29. On trouvera dans la relation B une description détaillée des objets disposés sur les « grèves des immortels », destinés à mettre en valeur les poèmes. 
Une fois les «grèves des immortels » présentées à l'empereur, les hauts dignitaires ont fait leur entrée, suivis des autres courtisans. C'est seulement après que tous les membres des équipes se furent installés à la place qui leur avait été assignée que les lecteurs des poèmes des deux équipes ont été appelés et que le concours proprement dit a commencé avec la lecture des pièces en lice et leur évaluation par l'arbitre. L'empereur poursuit :

Durant le concours, alcool et nourriture furent servis aux hauts dignitaires et aux membres de chaque équipe. Pendant la lecture des poèmes, l'équipe de gauche reprocha à l'équipe de droite d'avoir lu un poème sur les saules alors qu'il fallait lire deux poèmes sur la fauvette, ce qui entraîna la défaite [de l'équipe de droite]. Les vingt poèmes de chacune des équipes furent ainsi lus et confrontés. L'équipe de gauche remporta neuf victoires [et fut donc déclarée gagnante].

Le lecteur de l'équipe de droite aurait dû, à la troisième manche du concours, lire le second poème de son équipe sur la fauvette, mais déclama par erreur le poème sur les saules prévu pour la quatrième manche, suscitant ainsi une certaine confusion. L'empereur commet une erreur dans sa relation : comme l'atteste le procès-verbal du concours, l'équipe de gauche remporta onze victoires (et non neuf), l'équipe de droite quatre; il y eut cinq manches ex-сеquo :

Une fois la lecture des poèmes achevée, les musiciens de l'office de la musique furent appelés. Ils se répartirent entre la cour [close] Sud et la cour [close] Nord, et jouèrent des pièces à tour de rôle. Le ministre joua de la cithare et le grand conseiller Minamoto du luth. Pendant ce temps les coupes d'alcool circulèrent de nombreuses fois et les chants accompagnés par les instruments ne s'interrompirent jamais. Le ministre s'est levé de son siège et a offert de l'alcool. Alors que l'aube approchait, des présents furent faits aux participants selon leur rang, à commencer par le ministre. Le ministre reçut un ensemble de vêtements d'été à superposer ; le grand conseiller une tenue doublée blanche; les auditeurs une tenue non doublée blanche et les autres [participants] des coupons de soie.

La célébration s'est donc achevée par une distribution de récompenses aux différents participants, selon leur rang; les présents furent faits au nom de l'empereur, mais également de la part de la « dame du vestiaire » chef de l'équipe de gauche $^{30}$ :

30. La relation E nous apprend que la dame du vestiaire chef de l'équipe de gauche offrit une tenue complète au lecteur de son équipe. 
Une fois le jour paru, je me suis levé de mon fauteuil et j'ai gagné mes appartements. Mes serviteurs se sont [également] retirés. À l'aurore, du givre s'est déposé. Un de mes serviteurs proches me dit: «Il y a encore du givre et il fait si froid [alors que nous sommes en été]; les gens vont s'étonner de ces dérèglements du temps ».

Le 3 du quatrième mois, à l'heure du Mouton [vers 16 heures], la « grève des immortels » [de l'équipe de droite] du concours de poèmes fut portée à la Loge des Effluves parfumées [Higyôsha] et offerte à l'impératrice. Ensuite, quand les porteurs furent revenus, à l'heure du Coq [vers 18 heures], je leur fis porter la «grève des immortels » de l'équipe de gauche et son support chez la princesse Masako ${ }^{31}$.

C'est donc au lever du jour que l'empereur a quitté la salle et que la manifestation a officiellement - et réellement - pris fin. Deux jours après le concours, l'empereur fit porter une « grève des immortels » à son épouse l'impératrice, Fujiwara no Anshi (927-964), et une seconde «grève des immortels » à la princesse Masako, sa nièce ${ }^{32}$.

\section{Le sac aux savoirs poétiques}

Après avoir suivi le « Concours de Tentoku » dans son déroulement, examinons un autre document de toute première importance. Il s'agit du Fukurozôshi ou Sac [aux savoirs poétiques], traité de poésie compilé vers 1157 par Fujiwara no Kiyosuke ${ }^{33}$. Dans ce texte, Kiyosuke énumère, en se fondant sur des précédents, les vingt-cinq étapes du déroulement d'un concours de poèmes au palais : 1 . On décide à l'avance quels seront les thèmes des poèmes et l'on désigne le chef de chacune des équipes de gauche et de droite; 2 . Chaque équipe règle en concertation les détails qui la concernent (tenues des membres, "grèves des immortels », forme des baguettes de victoire, etc.) ; 3 . Prières et offrandes aux sanctuaires ${ }^{34}$; 4. Rituel de purification pour les hommes et les femmes de chacune des équipes ; 5. Aménagement de l'espace où se déroulera le concours ; 6. Les membres des équipes de gauche et de droite se rendent dans leur salle de réunion respective ; 7. À l'heure dite, l'empereur paraît ; 8. Les participants sont conviés : les hauts dignitaires, répartis en équipe de gauche et de droite, se placent devant l'empereur ; 9. L'équipe de gauche présente à l'empereur

31. Hagitani B. éd., Uta awase shû, p. 90.

32. La princesse Masako était la fille de l'empereur Suzaku (923-952), frère de l'empereur Murakami.

33. Édition utilisée : Fukurozôshi, éd. FuJIWARA T., coll. Shin Nihon Koten Bungaku Taikei, Tôkyô, vol.29, 1995. Signalons que nous traduisons le titre du traité suivant l'explication qui figure dans le colophon d'une copie de ce texte (cf. p. 170).

34. Pour remporter la victoire. 
un document sur lequel figurent, mis au net, la date du concours, les thèmes imposés et les poèmes produits par son équipe, puis l'équipe de droite fait de même ${ }^{35} ; 10$. Installation de la table présentoir pour les poèmes de l'équipe de gauche, ainsi que leurs «baguettes de victoire »; dans un second temps, l'équipe de droite fait de même ; 11. Une fois l'ordre donné, on apporte les lampes ; 12. On pose les coussins ronds pour les lecteurs et les personnes en charge des baguettes de victoire; 13. Les musiciens font leur entrée en jouant de la musique ${ }^{36} ; 14$. Les lecteurs des poèmes (kôji) et leurs adjoints (dokuji) ${ }^{37}$ sont appelés, les lecteurs en premier, puis les adjoints ; 15. On demande à l'arbitre de prendre place ; 16. Avant la déclamation des poèmes, on fait entrer une ou deux personnes reconnues dans leur domaine pour qu'elles donnent, si besoin est, leur avis ${ }^{38} ; 17$. Déclamation des poèmes ; 18. Évaluation des poèmes ; 19. Une fois la déclamation et l'évaluation des poèmes achevées, les deux lecteurs et les responsables des baguettes de victoire quittent leur place ; 20. Danse de l'équipe victorieuse ; 21. Salutations de l'équipe victorieuse ; 22. Alcool et nourriture offerts aux participants; 23. Divertissements musicaux; 24. Gratifications offertes aux ministres et autres fonctionnaires participants ; 25. Visite de l'équipe gagnante au sanctuaire pour remercier les dieux ${ }^{39}$.

Sur les vingt-cinq points énumérés par Kiyosuke, huit n'ont semblet-il pas été mis en œuvre dans le «Concours de Tentoku » : les points 3, 4 et 25 , en rapport avec la religion, ne sont pas mentionnés dans les relations de notre concours, mais peuvent s'être déroulées en dehors du cadre décrit par les documents dont nous disposons. Les points 9, 13 et 16 sont qualifiés par Kiyosuke de facultatifs et n'ont effectivement pas eu lieu lors de ce concours. Hagitani précise que les points 20 et 21 ne sont attestés que dans un seul concours respectivement : ils doivent donc également être considérés comme exceptionnels ${ }^{40}$. On peut ainsi noter qu'en dehors des points 3, 4 et 25 , en rapport avec la religion, le « Concours de Tentoku » comportait déjà toutes les parties jugées essentielles pour ce type de manifestations.

35. Le traité précise que dans certains cas ce document n'était pas présenté à l'empereur (cf. Fukurozôshi, p. 177).

36. Le traité précise que, dans certains cas, il n'y avait pas d'entrée en musique (cf. Fukurozôshi, p. 181).

37. Les adjoints (un par lecteur) sont chargés de transmettre au lecteur les poèmes qu'il doit déclamer.

38. Il semblerait que ce point n'ait été appliqué qu'exceptionnellement (un seul exemple attesté).

39. Fukurôzôshi, p. 173-186.

40. Hagitani B. éd., Uta awase shû, p. 18. 
Notre étude aura montré que les concours de poèmes organisés au palais impérial n'étaient pas de simples joutes poétiques. Le rituel, dans le sens d'organisation particulière ${ }^{41}$, dépassait très largement la simple mise en concours de poèmes. L'espace où se déroulait le concours, aménagé pour l'occasion, s'organisait autour de la personne du souverain. Les participants, choisis par l'empereur, prenaient place autour de lui dans un ordre strictement hiérarchisé et défini à l'avance. Takigawa Kôji souligne qu'une des fonctions de la poésie japonaise (waka) était de «donner une forme (keisei suru) », d'illustrer l'ordre qui régit les rapports entre le souverain et ses sujets ${ }^{42}$; il précise en outre que jusqu'à l'époque de l'empereur Murakami (celui qui ordonna le «Concours de Tentoku»), le pavillon de Pureté et de Fraîcheur où se déroula la manifestation était considéré comme un espace officiel (hare no $b a)^{43}$. Les manifestations qui s'y déroulaient étaient donc également considérées comme officielles.

Il ressort au terme de notre enquête que les concours de poèmes qui se déroulaient au palais impérial assumaient principalement trois fonctions : 1. confirmer le rôle de l'empereur comme souverain régnant sur ses sujets dans une parfaite harmonie, harmonie qu'illustrent à la fois la disposition de l'espace, la place des participants, la poésie produite et la musique jouée ; 2. confirmer la puissance matérielle du souverain en montrant des décors et des objets somptueux ; 3 . confirmer le rôle du souverain comme protecteur des arts. C'est donc afin de pouvoir assumer cette triple fonction que les concours de poèmes furent conçus comme de véritables rituels de cour.

\section{Michel Vieillard-Baron - Inalco/Centre d'Études Japonaises (CEJ)}

\section{Les concours de poèmes comme rituels de cour. Autour du « Concours de poèmes tenu au palais impérial la quatrième année de l'ère Tentoku [960] " (Tentoku yonen dairi uta awase)}

Les concours de poèmes furent pratiqués au Japon dès le IX siècle. Afin de déterminer les caractéristiques et la fonction de ces manifestations - notamment les plus officielles, celles qui se déroulaient au palais impérial nous examinons un exemple particulièrement significatif, le "Concours de poèmes tenu au palais impérial la quatrième année de l'ère Tentoku [960] " (Tentoku yonen dairi uta awase) organisé en 960 par l'empereur Murakami (926-967), et considéré comme un modèle du genre. Nous présentons tout d'abord le compte rendu de la manifestation rédigé par l'empereur Murakami en personne, puis, dans un second temps, les règles pour ce type de manifestation, telles que les a consignées le poète et poéticien Fujiwara no Kiyosuke (1104-1177) dans son important traité intitulé Fukurozôshi, le

41. Nous reprenons ici la définition de rituel proposée par M. Sot, L'Audience. Rituels et cadres spatiaux dans l'Antiquité et le haut Moyen Âge, Paris, 2007, p. 9.

42. Cf. Takigawa K., Tennô to bundan, Heian zenki no kôteki bungaku, Ôsaka, 2007, p. 18.

43. Ibid., p. 428. 
Sac [aux savoirs poétiques] texte compilé vers 1157. La confrontation de ces textes nous permettra de saisir comment se déroulaient ces rituels de cour et quelle était leur fonction.

Concours de poèmes (uta awase) - Fujiwara no Kiyosuke (1104-1177) Fukurozôshi (Sac [aux savoirs poétiques]) - Japon - rituels de cour

Poetry Competitions as Court Rituals. Form and Function of the "Imperial Palace Poetry Competition of the Fourth Year of Tentoku", Tentoku yonen dairi uta awase (960)

Poetry competitions took place in Japan from the ninth century onwards. In order to determine the characteristics and the functions of these competitions-in particular the most formal of them which took place in the Imperial Palace-I examine a particularly significant example, the "Imperial Palace Poetry Competition of the Fourth Year of Tentoku" (Tentoku yonen dairi uta awase) ordered in 960 by Emperor Murakami (926-967), and considered to be a model for poetry competition. First, I present the report of the poetry competition written by Emperor Murakami himself, then, I compare the imperial report to the rules for these competitions as the poet and poetic theorist Fujiwara no Kiyosuke (1104-1177) wrote them in his treaty Fukurozōshi, The Bag [of Poetic Knowledge] (ca 1157). Addressing these texts allows us to understand how these competitions, considered as court rituals, took place and what their function was.

Court Rituals - Fujiwara no Kiyosuke (1104-1177) - Fukurozōshi (The Bag [of Poetic Knowledge]) - Japan - Poetry Competions (uta awase) 
